\title{
Evaluation of index of cardiac electrophysiological balance in COVID-19 patients
}

\author{
Asoglu $\mathrm{R}^{1}$, Tibilli $\mathrm{H}^{1}$, Asoglu $\mathrm{E}^{2}$, Aladag $\mathrm{N}^{3}$, Ozdemir $\mathrm{M}^{4}$, Suner $\mathrm{A}^{1}$ \\ Adiyaman University Training and Research Hospital, Cardiology Department, Adiyaman, Turkey. \\ dr.asoglu@yahoo.com
}

\begin{abstract}
AIM: The aim of the current study was to evaluate the index of Cardiac Electrophysiological Balance (iCEB) in hospitalized COVID-19 patients receiving Hydroxychloroquine / azithromycin (HCQ / AZ) combination therapy to determine the susceptibility to ventricular arrhythmia among these patients.

METHOD: Sixty-seven COVID-19 patients admitted to the ward were included in the study.

Electrocardiograms (ECGs) were obtained from all patients before the initiation of treatment and on treatment day 5. QT/QRS (iCEB) and QTC/QRS (iCEBc) ratios were calculated.

RESULTS: QRS, QT and QTC intervals were significantly prolonged on day 5 measurements compared to pre-treatment period $(p<0.05)$. Overall, mean iCEB was $3.6 \pm 0.4$ before treatment and $3.8 \pm 0.4$ on day 5 in the study population $(p<0.001)$. Considering the iCEBc values, a significant increase was observed in patients receiving HCQ/AZ treatment compared to pre-treatment period $(4.1 \pm 0.5$ vs $4.4 \pm 0.6 ; p<0.001)$. CONCLUSIONS: To the best of our knowledge, this was the first study to investigate iCEB and iCEBC parameters in patients with COVID-19 on HCQ/AZ therapy. In this study, we demonstrated significantly increased iCEB and iCEBc values following HCQ/AZ treatment in COVID-19 patients. iCEB and iCEBC may serve as a noninvasive, simple, and novel biomarker for detecting increased pro-arrhythmia risk in COVID-19 patients (Tab. 3, Fig. 3, Ref. 36). Text in PDF www.elis.sk

KEY WORDS: COVID-19, index of Cardiac Electrophysiological Balance (iCEB), Hydroxychloroquine, azithromycin, ventricular arrhythmia.
\end{abstract}

\section{Introduction}

The severe acute respiratory syndrome coronavirus 2 (SARSCoV-2), commonly known as COVID-19, has become a global pandemic. COVID-19 causes clinical manifestations associated with severe acute respiratory distress syndrome (ARDS) (1). Several therapeutic strategies are currently investigated for the management of the COVID-19 pandemic. Hydroxychloroquine (HCQ), either as monotherapy or in combination with azithromycin (AZ), is a promising treatment option for symptomatic COVID-19 patients (2). Moreover, HCQ/AZ combination therapy has become one of the most commonly used treatment modalities in patients with moderate-to-severe COVID-19. The synergistic effect of $\mathrm{HCQ} / \mathrm{AZ}$ combination results from the actions of these two

\footnotetext{
${ }^{1}$ Adiyaman University Training and Research Hospital, Cardiology Department, Adiyaman, Turkey, ${ }^{2}$ Mardin Community Hospital, Cardiology Department, Mardin, Turkey, ${ }^{3}$ Yuzunci Yil University, Faculty of Medicine, Department of Cardiology, Van, Turkey, and ${ }^{4}$ Bayrampasa Kolan Hospital, Cardiology Department, Istanbul, Turkey
}

Address for correspondence: R. Asoglu, Dr, Adiyaman University Training and Research Hospital

Cardiology Department, Adiyaman, Turkey.

Phone: +905307763712, Fax: +904162233842 drugs on different binding sites on SARS-CoV-2 and AZ therapy enhances the effectiveness of HCQ. AZ is directed against the virus, whereas HCQ is directed against cellular adhesion cofactors (3). However, HCQ and AZ drugs block potassium channels and therefore, they may potentially prolong the QT interval $(4,5)$. Ventricular arrhythmias may occur due to QT interval prolongation induced by HCQ and AZ, and cases of torsades de pointes (TdP) have been reported with both drugs $(6,7)$. QT prolongation is more likely to occur in patients admitted to coronary intensive care unit, those with electrolyte disturbances and those taking other drugs that can prolong QT interval. The American Heart Association (AHA) guidelines recommend electrocardiographic (ECG) monitoring to detect QT prolongation in COVID-19 patients treated with HCQ and HCQ/AZ (8).

The QT interval is the most widely used ECG parameter to detect ventricular arrhythmias (9). The index of Cardiac Electrophysiological Balance (iCEB) is a novel noninvasive marker and reflects the balance between depolarization and repolarization of the cardiac action potential. iCEB is calculated by dividing QT interval by QRS duration (QT/QRS). In animal models, iCEB was shown to be a strong predictor of drug-induced cardiac arrhythmias (10). Robyns et al showed that iCEB can be used to detect increased arrhythmic risk in patients with supraventricular tachycardia receiving sotalol and flecainide. They also reported that 
while increased iCEB predisposes to torsades de pointes (TdP), decreased iCEB confers susceptibility to non-TdP mediated ventricular tachycardia (VT)/ventricular fibrillation (11).

Both HCQ and AZ are classified as drugs that are associated with a known risk of TdP (crediblemeds.org). HCQ/AZ combination therapy used for the treatment of COVID-19 may increase the iCEB value by causing QT prolongation. Thus, these drugs may have an indirect effect on the iCEB parameter. The aim of the current study was to evaluate iCEB in hospitalized COVID-19 patients on $\mathrm{HCQ} / \mathrm{AZ}$ combination therapy to determine the susceptibility to ventricular arrhythmia among these patients. This is the first study in the literature to assess iCEB in COVID-19 patients receiving $\mathrm{HCQ} / \mathrm{AZ}$ treatment.

\section{Materials and methods}

This study was designed as a retrospective and observational cohort study. Sixty-seven COVID-19 patients hospitalized in the ward between June 2020 and July 2020 were enrolled in the study. According to the World Health Organization (WHO) recommendation, the diagnosis was confirmed through the identification of the specific viral nucleic acid on nasal and pharyngeal swabs (real-time fluorescent, RT-PCR). Electrocardiograms (ECGs) were obtained from all patients before the initiation of treatment and on day 5. HCQ was given orally at a dose of $400 \mathrm{mg}$ BID for one day (loading dose) followed by $200 \mathrm{mg}$ BID for 4 days. Azithromycin was given orally at a dose of $500 \mathrm{mg}$ daily for 5 days. Exclusion criteria were as follows: drug allergy, severe electrolyte imbalance, chronic kidney failure, chronic liver failure, malignancies or inflammatory diseases, cardiac pacemakers, atrial fibrillation, atrial flutter, severe structural heart disease (left ventricular ejection fraction [ejection fraction] $\leq 30 \%$ ), presence of other ECG abnormality such as left ventricular hypertrophy, bifascicular block, complete left bundle branch block, second or third degree atrioventricular block, or pathological Q waves.

Drugs that could potentially cause QT prolongation were discontinued before initiation of treatment. Patients' demographics, clinical history, and medications were obtained from detailed chart review. Laboratory findings were retrieved from the hospital database. Hypertension (HT) was defined as a systolic blood pressure $\geq 140 \mathrm{mmHg}$, a diastolic blood pressure $\geq 90 \mathrm{mmHg}$, or requirement for antihypertensive medication. Type 2 diabetes mellitus (DM) was diagnosed according to the American Diabetes Association criteria. Written informed consent was obtained from all patients before enrollment in the study. This study was performed in accordance with the principles set forth in the Declaration of Helsinki and approved by the local Ethics Committee of our institution.

ECG recordings were obtained using standard 12-lead ECG. 12-lead ECG recordings (ECG 2550, Nihon Kohden, Japan) were performed after $30 \mathrm{~min}$ of rest, at room temperature. All ECGs (filter range $0.5-150 \mathrm{~Hz}, \mathrm{AC}$ filter $60 \mathrm{~Hz}, 25 \mathrm{~mm} / \mathrm{s}, 10 \mathrm{~mm} / \mathrm{mV}$ ) were reviewed by two independent clinicians who were blinded to the study design and clinical data. During the ECG recordings, all of the participants were in sinus rhythm. Resting heart rate was measured using the ECG data. All ECG recordings were scanned and the data was transferred to a personal computer to reduce possible errors. Adobe Photoshop software was used for $400 \%$ magnification. The measurements were performed on lead II and lead V5, and the longest QT interval was used for the analyses.

The QT interval was measured from the beginning of the QRS complex to the end of the T wave, and the QT interval was corrected for heart rate using the Bazett formula, QTc = QT $\sqrt{ }$ (R-R interval). The QRS duration was the interval between the first deflection of the QRS complex and the returning point to the baseline. The RR interval was measured as the distance between two consecutive $\mathrm{R}$ waves. Using these measurements, QT/QRS (iCEB) and QTc/QRS (iCEBc) ratios were calculated. Prolonged QTc was defined as an increase in QTc intervals of more than 60 milliseconds ( $\triangle \mathrm{QTc}>60$ milliseconds) compared with baseline or as a QTc of 500 milliseconds or greater (4). Continuous telemetry monitoring was used for patients with a QTc greater than 470 msec. In patients with a QTc prolongation of $>500 \mathrm{msec}$, reduced doses were administered or treatment was stopped. Patients with a baseline QTc greater than $500 \mathrm{msec}$ were not started on treatment. Ejection fraction $(\mathrm{EF})$ values were obtained before treatment from all patients at the time of admission using echocardiography. Hypokalemia and hypomagnesemia are associated with increased risk of serious arrhythmia. Therefore, correction of hypokalemia to a level $>4 \mathrm{mEq} / \mathrm{L}$ and hypomagnesemia to a level of $>2 \mathrm{mg} / \mathrm{dL}$ was performed when necessary.

\section{Statistical analyses}

Data were analyzed using the SPSS software version 25.0 for Windows (SPSS Inc, Chicago, Illinois). The Kolmogorov-Smirnov test was used to verify that continuous variables were normally distributed. Normally distributed variables were expressed as mean \pm standard deviation (SD), while non-normally distributed variables were expressed as median with interquartile range (IQR). The categorical variables were presented as percentages. Differences between two groups were analyzed using the Student's unpaired t-test or the Mann-Whitney U test for the parameters with a normal or non-normal distribution. The paired t-test and Wilcoxon signed-rank test was used to detect differences between paired measurements. The frequencies of nominal variables were compared using the Fisher's exact test or chi-square test. Statistical significance was defined as $\mathrm{p}<0.05$.

\section{Results}

Demographic and clinical characteristics of the study patients are shown in Table 1. The mean age of the participants was 45.3 \pm 17.3 years and $65 \%$ were male $(n=44)$. Regarding comorbidities of the patients, the rates of HT, DM, coronary artery disease and chronic obstructive pulmonary disease were low $(<20 \%)$. Corticosteroid therapy was administered to $25 \%$ of the patients during hospitalization. On biochemical analyses, sodium, potassium and creatinine values were within normal range. However, ferritin, C-reactive protein and troponin values were significantly elevated. Hematologic analysis showed normal white blood cell 
Tab. 1. The demographic and clinical data of the study population.

\begin{tabular}{lc}
\hline Age (years) & $45.3 \pm 17.3$ \\
Male n (\%) & $44(65)$ \\
Hypertension n (\%) & $12(18)$ \\
Diabetes mellitus n (\%) & $7(10)$ \\
Coronary artery disease n (\%) & $5(7)$ \\
COPD n (\%) & $6(9)$ \\
Sodium (mEq/L) & $138.8 \pm 3.1$ \\
Potassium (mEq/L) & $4.3 \pm 0.4$ \\
Creatine (mg/dl) & $0.9 \pm 0.2$ \\
White blood cell count $\left(10^{3} / \mathrm{mm}^{3}\right)$ & $7.3 \pm 3.2$ \\
Hemoglobin (g/dL) & $14.6 \pm 2.1$ \\
Platelet count (10 $\left.{ }^{3} / \mathrm{mm}^{3}\right)$ & $230.0 \pm 83.0$ \\
D-Dimer (ng/mL) & $380(610-1280)$ \\
Ferritin (ng/mL) & $189(78-605)$ \\
C-reactive protein (mg/L) & $21(6-74)$ \\
Troponin (ng/mL) & $2(1-5)$ \\
ACE inhibitors/ARB n $(\%)$ & $11(16)$ \\
Dihydropyridine CCB n $(\%)$ & $4(6)$ \\
Corticosteroid n $(\%)$ & $17(25)$ \\
\hline
\end{tabular}

COPD - Chronic obstructive pulmonary disease; ACE - Angiotensin-converting enzyme; ARB - Angiotensin receptor blocker; CCB - Calcium channel blocker.

count, hemoglobin and platelet count but increased D-Dimer levels (380[610-1280] ng/mL).

ECG data of all patients are shown in Table 2. No significant difference was detected in heart rate measurements on day 5 compared to baseline ( $p>.05$ ). However, QRS, QT and QTc were significantly increased on day 5 versus pre-treatment period $(\mathrm{p}<0.05)$. Overall, the mean $\mathrm{iCEB}$ value was $3.6 \pm 0.4$ at baseline and $3.8 \pm 0.4$ on treatment day $5(\mathrm{p}<0.001)$. There was a significant increase in $\mathrm{iCEBc}$ values in patients receiving $\mathrm{HCQ} /$ AZ treatment compared to pre-treatment $(4.1 \pm 0.5$ vs $4.4 \pm 0.6$; $p$ $<0.001$ ) (Fig. 1). At the end of 5 days, $\Delta \mathrm{QT}$ and $\Delta \mathrm{QTc}$ values were $20(10-40)$ and $23(8-45) \mathrm{msec}$, respectively, in patients receiving $\mathrm{HCQ} / \mathrm{AZ}$ treatment.

Tab. 2. Electrocardiographic data of the study population.

\begin{tabular}{lccc}
\hline & Admission & Fifth day & $p$-value \\
\hline Heart rate $(\mathrm{bpm})$ & $82.0 \pm 16.3$ & $79.7 \pm 17.2$ & 0.19 \\
QRS $(\mathrm{ms})$ & $100.0 \pm 8.7$ & $101.2 \pm 9.9$ & 0.02 \\
QT $(\mathrm{ms})$ & $355.5 \pm 36.9$ & $383.7 \pm 41.5$ & $<0.001$ \\
QTc $(\mathrm{ms})$ & $409.9 \pm 21.2$ & $436.6 \pm 33.0$ & $<0.001$ \\
iCEB & $3.6 \pm 0.4$ & $3.8 \pm 0.4$ & $<0.001$ \\
iCEBc & $4.1 \pm 0.5$ & $4.4 \pm 0.6$ & $<0.001$ \\
$\Delta$ QT $(\mathrm{ms})$ & & $20(10-40)$ & \\
$\Delta$ QTc $(\mathrm{ms})$ & $23(8-45)$ & \\
iCEB $-\mathrm{QT} / \mathrm{QRS} ;$ iCEBc $-\mathrm{QTc} / \mathrm{QRS} ; \Delta \mathrm{QT}-$ change in QT interval; $\Delta \mathrm{QTc}-$ change \\
in corrected QT interval.
\end{tabular}

Tab. 3. Electrocardiographic data of the female and male patients.

\begin{tabular}{|c|c|c|c|c|c|c|}
\hline & \multicolumn{3}{|c|}{ Female } & \multicolumn{3}{|c|}{ Male } \\
\hline & Admission & Fifth day & $\mathrm{p}$ & Admission & Fifth day & $\mathrm{p}$ \\
\hline Heart rate (bpm) & $86.0 \pm 15.3$ & $81.8 \pm 20.5$ & 0.17 & $79.9 \pm 16.6$ & $78.6 \pm 15.3$ & 0.55 \\
\hline QRS (ms) & $98.8 \pm 8.2$ & $100.3 \pm 10.0$ & 0.14 & $100.6 \pm 9.0$ & $101.7 \pm 9.9$ & 0.09 \\
\hline QT (ms) & $347.0 \pm 34.3$ & $382.6 \pm 37.9$ & $<0.001$ & $360.0 \pm 37.7$ & $384.3 \pm 43.6$ & $<0.001$ \\
\hline QTc (ms) & $411.2 \pm 24.0$ & $440.7 \pm 31.0$ & $<0.001$ & $409.3 \pm 19.8$ & $434.5 \pm 34.1$ & $<0.001$ \\
\hline iCEB & $3.5 \pm 0.4$ & $3.8 \pm 0.5$ & $<0.001$ & $3.6 \pm 0.4$ & $3.8 \pm 0.4$ & $<0.001$ \\
\hline $\mathrm{iCEBc}$ & $4.2 \pm 0.5$ & $4.5 \pm 0.7$ & $<0.001$ & $4.1 \pm 0.5$ & $4.3 \pm 0.6$ & $<0.001$ \\
\hline
\end{tabular}

iCEB - QT/QRS; iCEBc-QTc/QRS

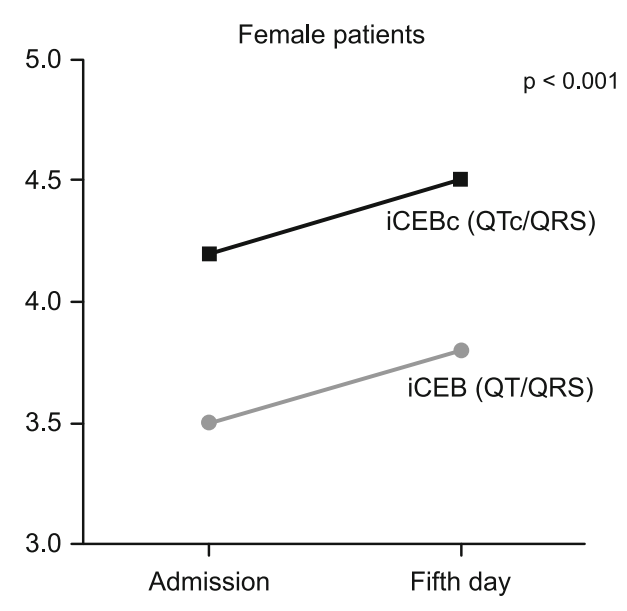

Fig. 2. iCEB and iCEBc measurements of female patients at baseline and on treatment day 5 .

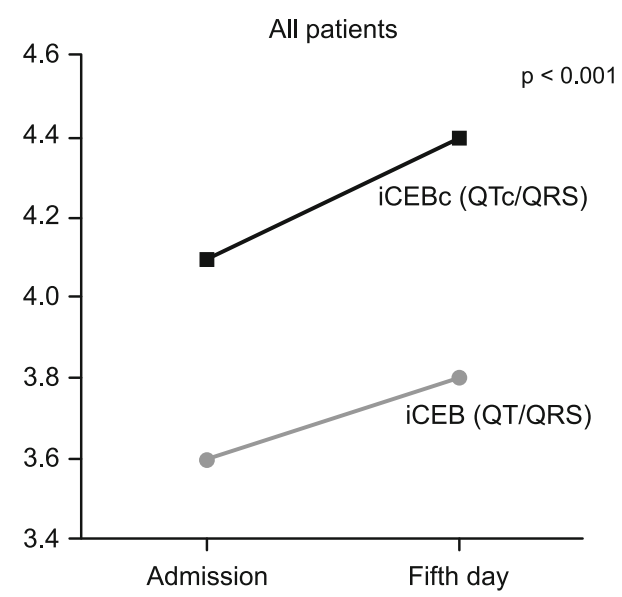

Fig. 1. The index of Cardiac Electrophysiological Balance (iCEB) and iCEBc values at baseline and on treatment day 5 for all patients.

A comparison of ECG changes after HCQ/AZ treatment between females and males is shown in Table 3. ECG recordings on day 5 showed that there was no significant change in heart rate and QRS but QT and QTc were significantly increased in both female and male patients. Increases in QT and QTc were greater in female patients than in male patients. $\mathrm{iCEB}$ and $\mathrm{iCEBc}$ values were also increased on day 5 versus pre-treatment in both female and male patients $(\mathrm{p}<0.001)$. Female patients showed greater increases in $\mathrm{iCEB}$ and $\mathrm{iCEBc}$ values compared to male patients (Fig 2 and 3). Despite these findings, none of the patients experienced arrhythmic events.

\section{Discussion}

In this study, we showed significant increases in iCEB and $\mathrm{iCEBc}$ values, reflecting predisposition to ventricular arrhythmia, in COVID-19 patients receiving HCQ/AZ 


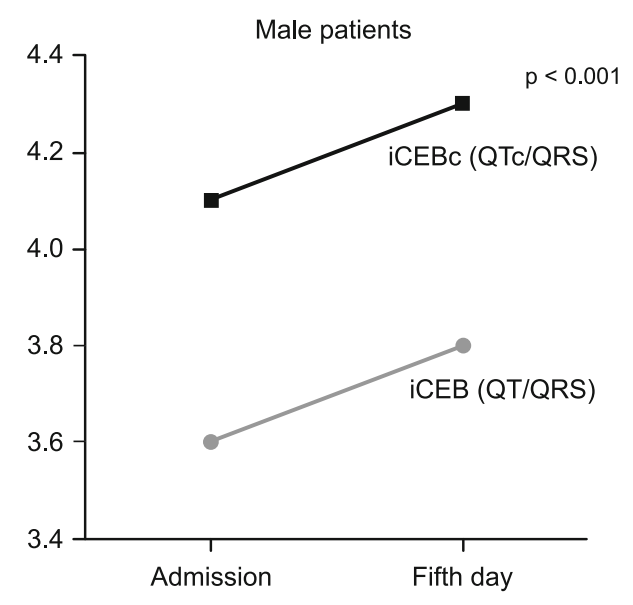

Fig. 3. iCEB and iCEBc measurements of male patients at baseline and on treatment day 5 .

treatment. Thus, based on increased iCEB values, we were able to demonstrate that COVID-19 patients on HCQ/AZ combination therapy were more susceptible to ventricular arrhythmia and particularly TdP. Furthermore, greater increases in iCEB and iCEBc values among female patients than in male patients suggest that $\mathrm{HCQ} / \mathrm{AZ}$ treatment confers a greater risk of arrhythmia in female patients.

iCEB is a simple, cost-effective, new and easily measured non-invasive biomarker to predict drug-induced cardiac arrhythmias than the current biomarkers (i.e., T-wave transmural dispersion and QT interval instability). The $\mathrm{iCEB}$ value derived from ECG is equivalent to the cardiac wavelength $\lambda[\lambda=$ Effective refractory period (ERP) x conduction velocity (CV)]) value obtained in an invasive electrophysiology laboratory. ERP represents changes in QT, whereas CV represent changes in QRS (10). Thus, iCEB can provide information about both the depolarization and repolarization phases of the cardiac action potential. Changes in iCEB may reflect an imbalance in electrophysiology and are thought to predict arrhythmias in correlation with $\lambda$. Arrhythmias predicted by iCEB include TdP and non-TdP-like ventricular tachycardia (VT) / ventricular fibrillation (VF). The main potential benefit of iCEB is the detection of both increased risk for TdP and non-TdP mediated VT/VF and therefore, iCEB might be a universal marker for ventricular arrhythmias. Antiarrhythmic drugs that shorten QRS interval or prolong QT interval are associated with an increase in iCEB and $\mathrm{iCEBc}$, hence an increased risk for TdP. In contrast, antiarrhythmic drugs that prolong QRS interval or shorten QT interval decrease iCEB and $\mathrm{iCEBc}$ and lead to an increased risk for non-TdP-like VT/VF.

Drug-induced long QT and its risk for TdP are now wellknown. Drugs that only slightly increase or decrease iCEB may be safe, while drugs markedly increasing or decreasing iCEB could potentially be pro-arrhythmic. However, large changes in the depolarization or repolarization of the action potential significantly change iCEB and may result in cardiac arrhythmic conditions. There is no standard cutoff value established for iCEB and studies should be conducted to determine this value. Nevertheless, a previous study identified the normal iCEB value as $4.24 \pm 5.24$. Studies in animal models have demonstrated that iCEB is a potential marker to predict drug-induced arrhythmias (10). A former study suggested that iCEB may offer a non-invasive and readily measurable marker to detect increased arrhythmic risk in patients with long QT syndrome (LQTS) or Brugada syndrome (11). Another study showed that the administration of dofetilide resulted in increased QT, Tp-e intervals and iCEB, causing TdP in rabbit ventricular wedge samples. In addition, they showed that encainide had no effect on QT and Tp-e intervals, reduced the iCEB values and resulted in non-TdP-like VT (10).

A previous study suggested that decreases in $\lambda$ by shortening the QT interval are related to the initiation and maintenance of reentry, which lead to re-entrant VT or fibrillation (12). Robyns et al suggested that sotalol administration increases iCEB, whereas flecainide usage decreases iCEB in patients with paroxysmal supraventricular arrhythmias. In the same study, the authors showed that compared to control group, $\mathrm{iCEB}$ was increased in patients with LQTS but decreased in patients with Brugada syndrome (11). In addition, it has been reported that iCEB was shortened due to prolonged QRS duration in patients with heart failure and patients with Brugada syndrome, which led to an increased risk of sudden cardiac death $(13,14)$. In our previous study, we evaluated $\mathrm{iCEB}$ and $\mathrm{iCEBc}$ in $\mathrm{AF}$ patients receiving propafenone and amiodarone. In that study, we observed the lowest iCEB among AF patients using propafenone compared to amiodarone users and control groups. Additionally, amiodarone group showed the highest $\mathrm{iCEBc}$ value and propafenone group showed the lowest iCEBc value (15).

Studies in different patient populations reported that iCEB indicates the susceptibility to developing malignant ventricular arrhythmia (15-17). Sivri et al assessed iCEB before and after hemodialysis in 52 patients with end-stage renal disease (ESRD). Before hemodialysis, ESRD group had a higher iCEB compared to control group. In addition, iCEB value increased after hemodialysis in ESRD patients and elevated iCEB was associated with an increased risk of TdP-mediated ventricular arrhythmia. Increases in $\mathrm{iCEB}$ and $\mathrm{iCEBc}$ after hemodialysis session resulted from QRS changes and no significant changes were reported for QT and QTc (16). Another contemporary study found that high iCEB values in the group undergoing imaging using computerized tomographic angiography due to suspected coronary artery disease were associated with increased pericardial fat volume (18). Yumurtaci et al evaluated iCEB as an indicator of ventricular arrhythmia in patients with acute myocarditis. The authors reported that iCEB values were significantly higher in the arrhythmia group compared to the non-arrhythmia group (17).

Hydroxychloroquine has been used for the treatment of rheumatic diseases and malaria for a long time and has been reported to be a potential therapeutic agent in the COVID-19 pandemic (19). HCQ inhibits viral infection by altering endosomal $\mathrm{pH}$ required for viral fusion and by affecting the glycosylation of host 
cell receptors by SARS-CoV-2 during its entry into the cell (20). In addition, in vitro studies have shown that HCQ effectively inhibits SARS-CoV-2 replication (21). The safety profile of HCQ is favorable, although cardiovascular side effects are known, including bradycardia, hypotension, refractory ventricular arrhythmia, QRS widening and QT interval lengthening (22). Since HCQ affects QT interval, it has a potential to cause TdP (23). The proposed arrhythmic mechanism is the blockade of the delayed rectifier potassium channel in the myocardial cells, which results in prolongation of ventricular repolarization and QT interval (24). This effect of hydroxychloroquine can be more pronounced when used with other drugs that prolong QT interval. Azithromycin is a macrolide antibiotic which is effective in ARDS (25). Cardiovascular effects of azithromycin have been investigated in previous studies (26). Azithromycin is known to induce QT prolongation, but its use is rarely associated with adverse cardiac events like ventricular arrhythmias. Azithromycin exerts is proarrhythmic effects by affecting cardiac sodium channels and increasing intracellular sodium concentration (27).

$\mathrm{HCQ} / \mathrm{AZ}$ combination may cause ventricular arrhythmias by prolonging the QT interval and concerns have been raised regarding the use of this combination due to arrhythmic potential. ECG monitoring is recommended to detect QT prolongation that may occur with the use of $\mathrm{HCQ} / \mathrm{AZ}$ combination. These two drugs are being widely used to treat COVID-19 despite the known risk of QT interval prolongation. It is important to determine the proarrhythmic risk and significant QTc-prolonging effect of $\mathrm{HCQ} / \mathrm{AZ}$ combination therapy in COVID-19 patients. In addition, guidelines recommend discontinuation of these drugs at a QTc $\geq 500 \mathrm{msec}$ or when QTc is prolonged by more than $60 \mathrm{msec}$ (28). Some publications in the literature have reported that HCQ/ AZ combination therapy prolongs QT duration in patients with COVID-19 (29). Moreover, HCQ/AZ combination results in a greater increase in QTc interval compared to chloroquine or HCQ monotherapy (30). Maraj et al showed that QTc prolongation was present in one in four hospitalized COVID-19 patients. Therefore, the authors suggested that HCQ/AZ combination therapy should not be administered without continuous telemetry monitoring. They also reported that older age, impaired renal function, baseline QTc $>460 \mathrm{msec}$, and concurrent use of other high-risk QTc-prolonging drugs were predictors of QTc prolongation (31). In a separate study, among 53 patients receiving HCQ/AZ combination therapy, 11 patients had a QT prolongation exceeding $500 \mathrm{msec}$ which led to cessation of treatment in the majority of these patients (32).

One study involving COVID-19 patients reported significant QTc prolongation in those receiving $\mathrm{HCQ} / \mathrm{AZ}$ combination therapy than patients receiving HCQ monotherapy alone or no treatment. Additionally, QTc prolongation was more pronounced in older COVID-19 patients receiving $\mathrm{HCQ} / \mathrm{AZ}[\mathrm{HCQ} / \mathrm{AZT}$ combination (OR 9.02, p =0.001) and older age (OR 1.04, p = $0.031)$ ] (33). However, since no arrhythmic fatalities occurred in patients given HCQ/AZ therapy, the use of such drugs may be considered as safe relating to arrhythmic risk in the treatment of COVID-19 patients. In a separate study, Saleh et al evaluated 280 patients with COVID-19 receiving monotherapy or combination therapy. They reported significantly longer QTc in patients treated with $\mathrm{HCQ} / \mathrm{AZ}$ than in patients receiving monotherapy but changes in QRS were comparable between the groups. No arrhythmic events were observed in patients treated with monotherapy or combination therapy (30). QTc prolongation also occurred in COVID-19 patients who were given a 5-day course of $\mathrm{HCQ} / \mathrm{AZ}$ combination therapy but maximal prolongation was $30 \mathrm{msec}$ (34). Contrastingly, Bakhshaliyev et al reported that $\mathrm{HCQ} / \mathrm{AZ}$ treatment administered for 5 days did not cause significant QT prolongation or conduction disorders in non-intensive care unit COVID-19 patients compared to baseline ECG parameters (35).

In the current study, we also evaluated electrocardiographic changes in patients with COVID-19 both, before treatment and on day 5. Significant differences were observed in QRS, QT, QTc, iCEB and iCEBc values on day 5 compared to pretreatment. When we investigated whether there was a sex-related difference in the changes in ECG parameters, female patients were found to have more pronounced increases in QT, QTc, iCEB and $\mathrm{iCEBc}$ than male patients. Based on these findings, we suggest that closer ECG monitoring is warranted for female COVID-19 patients receiving $\mathrm{HCQ} / \mathrm{AZ}$ combination therapy. Similarly, Grewal et al compared ECG parameters by gender in COVID-19 patients receiving either HCQ or $\mathrm{HCQ} / \mathrm{AZ}$. The authors reported significantly longer QTc intervals in female COVID-19 patients on HCQ or HCQ/AZ treatment than in males (36). In addition, confirmation of QTc prolongation in COVID-19 patients better informs physicians of this important side effect of HCQ/AZM that will necessitate QTc assessment prior to drug initiation, and QTc monitoring during treatment for many patients.

\section{Limitations}

This study has several limitations. The number of cases was small due to the single-center design of the study. The relatively small sample size could have limited the strength of the results as well as the conclusions that can be drawn from this work. All patients enrolled in the study were ward patients and exclusion of patients with moderate-to-severe ARDS hospitalized in the intensive care unit is another limitation of the study. An additional limitation is exclusion of COVID-19 patients receiving HCQ or AZ monotherapy. Since no deaths occurred in the patients followed during the study, the correlation between mortality and iCEB could not be examined. Also, the study did not involve Holter cardiac monitoring or an assessment of other ECG parameters such as Tp-e. In addition, it is possible that the COVID-19 infection could directly prolong the QTc independent of drug effects. However, despite these limitations, we believe that our research provides essential insights into the ECG changes and provides a significant baseline for future studies examining the association between iCEB and potential arrhythmia risk in COVID-19 patients on $\mathrm{HCQ} / \mathrm{AZ}$ therapy. 


\section{Conclusion}

To the best of our knowledge, this was the first study to investigate $\mathrm{iCEB}$ and $\mathrm{iCEBc}$ parameters in patients with COVID-19 on $\mathrm{HCQ} / \mathrm{AZ}$ therapy. Marked increases were found in $\mathrm{iCEB}$ and iCEBc values in COVID-19 patients receiving a 5-day course of $\mathrm{HCQ} / \mathrm{AZ}$ combination therapy, indicating susceptibility to ventricular arrhythmia. iCEB and iCEBc may serve as a noninvasive, simple, and novel biomarker for detecting increased pro-arrhythmia risk in COVID-19 patients. These findings suggest that ECG monitoring is required for COVID-19 patients throughout HCQ/ AZ treatment. Further studies are needed to determine whether these electrophysiological changes are associated with ventricular arrhythmias in COVID-19 patients on $\mathrm{HCQ} / \mathrm{AZ}$ therapy.

\section{References}

1. Wu Z, McGoogan JM. Characteristics of and important lessons from the coronavirus disease 2019 (COVID-19) outbreak in China: summary of a report of 72314 cases from the Chinese Center for Disease Control and Prevention. JAMA 2020; 323 (13): 1239-1242.

2. Kupferschmidt K, Cohen J. Race to find COVID-19 treatments accelerates 2020 .

3. Fantini J, Chahinian H, Yahi N. Synergistic antiviral effect of hydroxychloroquine and azithromycin in combination against SARS-CoV-2: What molecular dynamics studies of virus-host interactions reveal. Int J Antimicrob Agents 2020; 56 (2): 106020.

4. Drew B, Ackerman M, Funk M et al. American heart association acute cardiac care committee of the council on clinical cardiology, the council on cardiovascular nursing, and the American College of Cardiology Foundation. Prevention of torsade de pointes in hospital settings: a scientific statement from the American Heart Association and the American College of Cardiology Foundation. Circulation 2010; 121 (8): 1047-1060.

5. Yazdany J, Kim AH. Use of hydroxychloroquine and chloroquine during the COVID-19 pandemic: what every clinician should know 2020.

6. Chen C-Y, Wang F-L, Lin C-C. Chronic hydroxychloroquine use associated with QT prolongation and refractory ventricular arrhythmia. Clin Toxicol 2006; 44 (2): 173-175.

7. Choi Y, Lim H-S, Chung D, Choi J, Yoon D. Risk evaluation of azithromycin-induced QT prolongation in real-world practice. BioMed Res Int 2018; 2018.

8. Roden DM, Harrington RA, Poppas A, Russo AM. Considerations for drug interactions on QTc interval in exploratory COVID-19 treatment. J Am Coll Cardiol 2020; 75 (20): 2623-2624.

9. G Postema P, AM Wilde A. The measurement of the QT interval. Curr Cardiol Rev 2014; 10 (3): 287-294.

10. Lu HR, Yan G-X, Gallacher DJ. A new biomarker-index of Cardiac Electrophysiological Balance (iCEB) - plays an important role in druginduced cardiac arrhythmias: beyond QT-prolongation and Torsades de Pointes (TdPs). J Pharmacol Toxicol Methods 2013; 68 (2): 250-259.

11. Robyns T, Lu HR, Gallacher DJ et al. Evaluation of index of cardioelectrophysiological balance (iCEB) as a new biomarker for the identification of patients at increased arrhythmic risk. Ann Noninvasive Electrocardiol 2016; 21 (3): 294-304.
12. Aidonidis I, Poyatzi A, Stamatiou G, Lymberi M, Stamatoyannis N, Molyvdas P-A. Dose-related shortening of ventricular tachycardia cycle length after administration of the KATP channel opener bimakalim in a 4-day-old chronic infarct anesthetized pig model. J Cardiovasc Pharmacol Ther 2009; 14 (3): 222-230.

13. Kashani A, Barold SS. Significance of QRS complex duration in patients with heart failure. J Am Coll Cardiol 2005; 46 (12): 2183-2192.

14. Ohkubo K, Watanabe I, Okumura Y et al. Prolonged QRS duration in lead V2 and risk of life-threatening ventricular Arrhythmia in patients with Brugada syndrome. Int Heart J 2011; 52 (2): 98-102.

15. Afsin A, Asoglu R, Kobat MA, Asoglu E, Suner A. Evaluation of Index of Cardio-Electrophysiological Balance in Patients with Atrial Fibrillation on Antiarrhythmic-Drug Therapy. Cardiol Res 2021; 12 (1): 37 .

16. Sivri S, Çelik M. Evaluation of index of cardiac-electrophysiological balance before and after hemodialysis in patients with end-stage renal disease. J Electrocardiol 2019; 54: 72-75.

17. Yumurtaci O, Kurt C, Ucar M. O Cihan. Usefulness of Electrocardiographic Markers To Predict Ventricular Arrhythmias In Acute Myocarditis Patients. Turk Med Stud J 2017; 4: 6-10.

18. Nafakhi H, Al-Mosawi AA, Alareedh M, Al-Nafakh HA. Index of cardiac electrophysiological balance and transmural dispersion of the repolarization index relationships with pericardial fat volume and coronary calcification. Biomark Med 2018; 12 (4): 321-328.

19. Cortegiani A, Ingoglia G, Ippolito M, Giarratano A, Einav S. A systematic review on the efficacy and safety of chloroquine for the treatment of COVID-19 [published online March 10, 2020]. J Crit Care Doi. 10

20. Vincent M, Bergeron E, Benjannet S, Erickson B, Rollin P, Ksiazek T. Virol. Chloroquine Potent Inhib SARS Coronavirus Infect Spread J 2005; 2: 69 .

21. Yao X, Ye F, Zhang $M$ et al. In vitro antiviral activity and projection of optimized dosing design of hydroxychloroquine for the treatment of severe acute respiratory syndrome coronavirus 2 (SARS-CoV-2). Clin Infect Dis 2020; 71 (15): 732-739.

22. Khobragade SB, Gupta P, Gurav P, Chaudhari G, Gatne MM, Shingatgeri VM. Assessment of proarrhythmic activity of chloroquine in in vivo and ex vivo rabbit models. J Pharmacol Pharmacother 2013; 4 (2): 116.

23. Morgan ND, Patel SV, Dvorkina O. Suspected hydroxychloroquineassociated QT-interval prolongation in a patient with systemic lupus erythematosus. JCR J Clin Rheumatol 2013; 19 (5): 286-288.

24. World Health Organization. The cardiotoxicity of antimalarials. Geneva Switz WHOGoogle Sch 2017;

25. Kawamura K, Ichikado K, Takaki M, Eguchi Y, Anan K, Suga M. Adjunctive therapy with azithromycin for moderate and severe acute respiratory distress syndrome: a retrospective, propensity score-matching analysis of prospectively collected data at a single center. Int J Antimicrob Agents 2018; 51 (6): 918-924.

26. Gorelik E, Masarwa R, Perlman A, Rotshild V, Muszkat M, Matok I. Systematic review, meta-analysis, and network meta-analysis of the cardiovascular safety of macrolides. Antimicrob Agents Chemother 2018; 62 (6).

27. Yang Z, Prinsen JK, Bersell KR et al. Azithromycin causes a novel proarrhythmic syndrome. Circ Arrhythm Electrophysiol 2017; 10 (4): e003560. 


\section{$598-604$}

28. Giudicessi JR, Noseworthy PA, Friedman PA, Ackerman MJ. Urgent guidance for navigating and circumventing the QTc-prolonging and torsadogenic potential of possible pharmacotherapies for coronavirus disease 19 (COVID-19). In Elsevier; 2020. p. 1213-1221.

29. Bessière F, Roccia $\mathbf{H}$, Delinière $\mathbf{A}$ et al. Assessment of QT intervals in a case series of patients with coronavirus disease 2019 (COVID-19) infection treated with hydroxychloroquine alone or in combination with azithromycin in an intensive care unit. JAMA Cardiol 2020; 5 (9): 1067-1069.

30. Saleh M, Gabriels J, Chang D et al. Effect of chloroquine, hydroxychloroquine, and azithromycin on the corrected QT interval in patients with SARS-CoV-2 infection. Circ Arrhythm Electrophysiol 2020; 13 (6): e008662.

31. Maraj I, Hummel JP, Taoutel R et al. Incidence and determinants of QT interval prolongation in COVID-19 patients treated with hydroxychloroquine and azithromycin. J Cardiovasc Electrophysiol 2020; 31 (8): 1904-1907.

32. Mercuro NJ, Yen CF, Shim DJ et al. Risk of QT interval prolongation associated with use of hydroxychloroquine with or without con- comitant azithromycin among hospitalized patients testing positive for coronavirus disease 2019 (COVID-19). JAMA Cardiol 2020; 5 (9): 1036-1041.

33. Bernardini A, Ciconte G, Negro G et al. Assessing QT interval in COVID-19 patients: safety of hydroxychloroquine-azithromycin combination regimen. Int J Cardiol 2020.

34. O’Connell TF, Bradley CJ, Abbas AE et al. Hydroxychloroquine/ Azithromycin Therapy and QT Prolongation in Hospitalized Patients with COVID-19. JACC Clin Electrophysiol 2020.

35. Bakhshaliyev N, Uluganyan M, Enhos A, Karacop E, Ozdemir R. The effect of 5-day course of hydroxychloroquine and azithromycin combination on QT interval in non-ICU COVID19 (+) patients. J Electrocardiol 2020; 62: 59-64.

36. Grewal S, Jankelson L, Van Den Broek MP et al. QTc prolongation risk evaluation in Female COVID-19 patients undergoing chloroquine and hydroxychloroquine with/without azithromycin treatment. Front Cardiovasc Med 2020; 7: 152 . 